Supporting Information

\title{
Ligand-Dependent Coalescence Behaviors of Gold Nanoparticles Studied by Multichamber Graphene Liquid Cell Transmission Electron Microscopy
}

Yuna Bae ${ }^{\dagger,}$, Kitaek Lim ${ }^{\S}$, Seulwoo Kim ${ }^{\dagger}$, Dohun Kang ${ }^{\dagger, \star}$, Byung Hyo Kim ${ }^{\dagger, \star, \|, ~ J o o d e o k ~}$ $\mathrm{Kim}^{\dagger, \star}$, Sungsu Kang ${ }^{\dagger, \dagger}$, Sungho Jeon ${ }^{\S}$, JunBeom $\mathrm{Cho}^{\dagger}$, Won Bo Lee ${ }^{*}$, , Won Chul Lee ${ }^{*}$, , Jungwon Park ${ }^{*}, \dagger$,

†School of Chemical and Biological Engineering, and Institute of Chemical Processes, Seoul National University, Seoul 08826, Republic of Korea

Center for Nanoparticle Research, Institute of Basic Science (IBS), Seoul 08826, Republic of Korea

${ }^{\S}$ Department of Mechanical Engineering, Hanyang University, Ansan, Gyeonggi 15588, Republic of Korea

"Department of Organic Materials and Fiber Engineering, Soongsil University, Seoul 06978, Republic of Korea

*E-mail: jungwonpark@snu.ac.kr (J.P.); wonchullee@hanyang.ac.kr (W.C.L.); wblee@snu.ac.kr (W.B.L.) 


\section{Experimental details}

\subsection{Materials}

Gold(III) chloride trihydrate $\left(\mathrm{HAuCl}_{4}, \geq 99.9 \%\right.$, Aldrich), hexadecyltrimethylammonium bromide (CTAB, $\geq 98 \%$, Aldrich), n-octylamine (99\%, Aldrich), and ammonium persulfate $\left(\left(\mathrm{NH}_{4}\right)_{2} \mathrm{~S}_{2} \mathrm{O}_{8}, \geq 99.9 \%\right.$, Aldrich) were utilized as received. Ultrathin anodic aluminum oxide (AAO, TopMembranes Technology, Inc.) and Quantifoil® R 1.2/1.3 holey carbon TEM 200 mesh gold grids were also employed.

\subsection{Preparation of aqueous gold precursor solution}

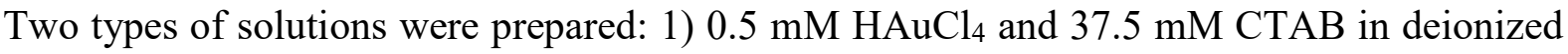
water and 2) $0.5 \mathrm{mM} \mathrm{HAuCl}_{4}, 37.5 \mathrm{mM} \mathrm{CTAB}$, and $12.1 \mathrm{mM}$-octylamine in deionized water.

\subsection{Preparation of multichamber graphene liquid cell}

The multichamber graphene liquid cells were fabricated based on previously reported methods. ${ }^{1}$ A porous AAO membrane with pore-diameter, interpore distance, and thickness of $\sim 80, \sim 125$, and $\sim 50 \mathrm{~nm}$, respectively, was employed in this study. The dimensions of the AAO membrane are selectively controllable by changing the anodization parameters, thus the liquid thickness of the liquid cell can be adjusted using the AAO membrane with a desired thickness suitable for the purpose. Graphene, for the fabrication of the liquid cell, was synthesized on a $25 \mu \mathrm{m}$ thick copper foil (99.8\%, Alfa Aesar) by the chemical vapor deposition (CVD) method. For the sample preparation, graphene was first transferred onto the Quantifoil ${ }^{\circledR}$ R 1.2/1.3 holey carbon TEM grids by direct transfer. ${ }^{2}$ Summarily, the grid was placed onto a graphene-coated copper foil with the Quantifoil carbon-film side facing graphene. Next, 2-propanol was dropped onto the sample to wet its interface, and the sample was subsequently dried on a hot plate for $5 \mathrm{~min}$, at $50{ }^{\circ} \mathrm{C}$. The grid was floated on a $10 \%$ aqueous solution of $\left(\mathrm{NH}_{4}\right)_{2} \mathrm{~S}_{2} \mathrm{O}_{8}$ to etch the underlying copper foil, after which it was rinsed severally by floating the graphene-transferred grid on deionized water, followed by drying, at $70{ }^{\circ} \mathrm{C}$, in an oven. The AAO membrane was placed onto the graphene-transferred grid, after which deionized water was dropped on it. Thereafter, it was dried in an oven for $5 \mathrm{~min}$, at $70{ }^{\circ} \mathrm{C}$, to bond the AAO membrane to the graphene layer. Next, the PMMA substrate, which was the supporting layer of the AAO membrane, was removed by immersing the assembled grid in an acetone bath. The resulting grid possessed nanoscale multiwells (graphene floor with AAO sidewalls). Afterward, $0.5 \mu \mathrm{L}$ of the prepared gold precursor solution was loaded on the AAO side of the prepared grid, after which another graphene-transferred grid was placed onto it. As the residual liquid samples were soaked and dried, the utilized liquid sample was encapsulated in the multichambers. 


\subsection{Acquisition of TEM images}

TEM observations of the gold nanoparticles in the multichamber graphene liquid cell were performed on a JEM-2100F (JEOL, Japan) instrument, operated at $200 \mathrm{kV}$, and equipped with an UltraScan 1000XP CCD detector (Gatan). Further, in situ TEM movies were recorded in $10 \mathrm{fps}$. The dose rate of the electron-beam was consistently maintained, at 4000-8000 $e^{-} \AA^{-2} \mathrm{~s}^{-1}$, which is the selected range to promote a condition where we can observe coalescence-induced growth of nanoparticles. In low electron-beam dose rates $(\sim 50$ and $\sim 300 e^{-} \AA^{-2} \mathbf{S}^{-1}$ ), the etching of gold nanoparticles is the dominant process, consistent with the previous report, ${ }^{3}$ as shown in Figure S11. (In situ TEM imaging under the low dose condition (Figure S2a) was performed on a JEM-ARM200F microscope (JEOL, Japan) installed at National Center for Inter-University Research Facility (Seoul National University, Seoul, Korea). The microscope was equipped with an aberration corrector in the objective lens and an OneView IS camera (Gatan, USA) and operated at $200 \mathrm{kV}$. The in situ TEM movies were recorded in $12.5 \mathrm{fps}$.)

\subsection{Quantitative analysis of nanoparticle coalescence}

Employing a homemade Matlab algorithm, the observed nanoparticles inside the multichambers were distinguished from the background. The algorithm reduced the Gaussian noise, employing Gaussian and Weiner filters and thereafter, a high-contrast change, at the edges of the nanoparticles, were emphasized through Laplacian filtering. Thereafter, the images were binarized by the adaptive thresholding method. We selected the dark areas to represent the nanoparticles and tracked the nanoparticles, which underwent coalescence.

We measured various parameters for all the tracked nanoparticles from the time-series of binarized images; we measured the distance between the surfaces of the approaching nanoparticle pair, the end-to-end distance along the long axis of the pair, the projected nanoparticle area, and the perimeter of the merged nanoparticle. We calculated the circularity $(C)$ of the merged nanoparticles by the following equation:

$$
C=4 \pi A / P^{2},
$$

where $A$ is the projected area of the merged nanoparticle and $P$ is the perimeter of the merged nanoparticle. 


\subsection{MD simulations details}

The MD simulations were performed to calculate the atomic local environment and microscopic dynamics of the CTAB bilayers, at various concentrations of n-octylamine, on the gold nanoparticles. There are many approaches to obtain the parameters for the description of the molecular interactions. In this study, we selected the force-field, based on CHARMM $36,{ }^{3}$ because of its good agreement with the experimental results. Moreover, it served the parameter sets for specific molecular groups, such as proteins, lipids, and metals. Storm et al. developed the force-field parameters for the CTAB molecules from other related lipid compounds ${ }^{4}$, and we adopted their method. The n-octylamine ligand was obtained similarly, from a neutral lysine molecule. The TIP3P model was employed to describe the water molecules, and the parameters for the gold atoms were obtained from the CHARMMMETAL force-field set. To elucidate the effect of $n$-octylamine on the CTAB bilayer in the gold nanoparticles, we located the $2 \mathrm{D}$ infinite gold nanorods on the simulation box, under periodic boundary conditions ( $41 \AA \times 41 \AA \times 150 \AA$ ) to form the (100), (110), and (111) FCC surfaces. Further, the CTAB bilayers and n-octylamine were each placed below the surface. The system without n-octylamine was composed of 212 CTAB molecules, and we replaced some of the CTAB molecules with n-octylamine molecules. We denoted the systems with various concentrations as $\mathrm{C}_{x} \mathrm{O}_{y}(x=100,75,67,50$, and $y=0,25,33,50)$, where $\mathrm{C}$ is CTAB and $\mathrm{O}$ is n-octylamine.

The systems were equilibrated in the NpT ensemble for $10 \mathrm{~ns}$, at $300 \mathrm{~K}$, and $1 \mathrm{bar}$, after the energy minimization procedure, employing the PPPM technique. ${ }^{5}$ All the data utilized in this study were collected after another $10 \mathrm{~ns}$ of simulation, under the same conditions. All the bonds related to hydrogen were constrained by the SHAKE algorithm. ${ }^{6}$ MD simulations and calculation of the local density and mean-square displacement (MSD) were performed by the LAMMPS open-source software. ${ }^{7}$ We checked the results by changing the initial composition of the ligand shell (location of octylamine and CTAB molecules). The initial configuration of the ligand shells does not have significant effect on their calculation (final structure and ligand mobility), and the mixing ratio of the ligand shells seems to be a more important factor determining their characteristics. 
1.7. Liquid thickness measurement of the multichamber liquid cell

We measure the liquid thickness encapsulated in the multichamber liquid cell using the cryoelectron energy loss spectroscopy (cryo-EELS) analysis. The procedure is to record unfiltered and zero-loss filtered images of a given sample site and to calculate the logarithm of the intensity ratio between the area $I_{0}$ under the zero-loss peak and the total area $I_{t}$ under the whole spectrum. ${ }^{9}$ The thickness $t$ is given by

$$
\frac{t}{\lambda}=-\ln \left(\frac{I_{0}}{I_{t}}\right)
$$

where $\lambda$ is the total inelastic mean free path of an electron. In the field of cryo-EM, many papers applied this measurement to obtain thickness of the vitreous ice. ${ }^{10-12}$ The thickness of vitreous ice are measured through the EELS measurement in cryogenic state to prevent the evaporation of encapsulated liquid caused by electron-beam damage of graphene windows. The inelastic mean free path of an electron in vitreous ice is calculated as $287 \mathrm{~nm}$ at $200 \mathrm{kV}$. The average ice thickness of 22 independent chambers with a $60-\mathrm{nm}$ thick AAO membrane is $39.8 \pm 11.5 \mathrm{~nm}$. An exemplary STEM image of the multichamber liquid cell and the measured ice thicknesses are presented in Figure S1. 
1.8. Discussion for the anomalous motion of nanoparticles in multichamber liquid cells

As previously reported observations, nanoparticles in multichamber liquid cells also exhibit retarded diffusion behaviors, with diffusion coefficient of $\sim 10^{-3}$ to $10^{-1} \mathrm{~nm}^{2} / \mathrm{s}$, which is 7 to 9 orders of magnitude slower than the expected values for a bulk solution. ${ }^{13-16}$ The reason of this anomalous motion of nanoparticles observed in liquid-phase TEM still need to be studied. The incident electrons can cause unwanted effects on liquid sample including radiolysis, chemical perturbations, and secondary charging, which possibly influence the behavior of diffusing nanoparticles. ${ }^{3,17}$ The use of graphene as a window material has been reported to prevent those detrimental effects during in situ TEM imaging, such as scavenging generated radicals and minimizing electron-beam damage. ${ }^{18,19}$ Other sources that we should consider include the confinement effect and surface interaction with window materials (graphene and $\mathrm{SiN}_{\mathrm{x}}$ ) of nanoparticles or capping ligands. In addition to these, we also suppose that there might be direction convection of solvent in the nano-sized chamber during imaging which could abnormally affect diffusion dynamics of encapsulated nanoparticles. Those various effects could occur in the multichamber graphene liquid cells, and further work should be required to fully address how they occur and how they affect. Nevertheless, during the coalescence process, desorption or movement of ligands trapped in the inter-particle space is necessarily involved, and this step is probably closely related to the mobility of ligands. In particular, even if there is an interaction between graphene and capping ligands, it is mainly contributed by the exposed functional groups of the ligand shell toward graphene which are presumably similar for the two control cases since the outermost region of the ligand shell in both cases are mainly composed of CTAB. It is also notable that trajectories of nanoparticles in the current study does not show a typical stick-slip motion that are observed in the liquid phase TEM when there is a strong interaction between nanoparticles and the substrate surface. 


\section{Supporting movie captions}

Movies S1. In situ liquid-phase TEM movie of the gold nanoparticles capped with the CTAB ligands inside a multichamber. The movie plays four times faster than in real-time (scale bar, $20 \mathrm{~nm})$.

Movies S2. In situ liquid-phase TEM movie of the gold nanoparticles capped with a mixed ligands inside a multichamber. The movie plays four times faster than in real-time (scale bar, $20 \mathrm{~nm})$.

Movies S3. In situ liquid-phase TEM movie of the coalescences of 41 pairs in the AuNPs/CTAB system. The movie plays two times faster than in real-time (scale bar, $5 \mathrm{~nm}$ ).

Movies S4. In situ liquid-phase TEM movie of the coalescence of 45 pairs in the AuNPs/Mixed system. The movie plays two times faster than in real-time (scale bar, $5 \mathrm{~nm}$ ). 


\section{Supporting figures and tables}

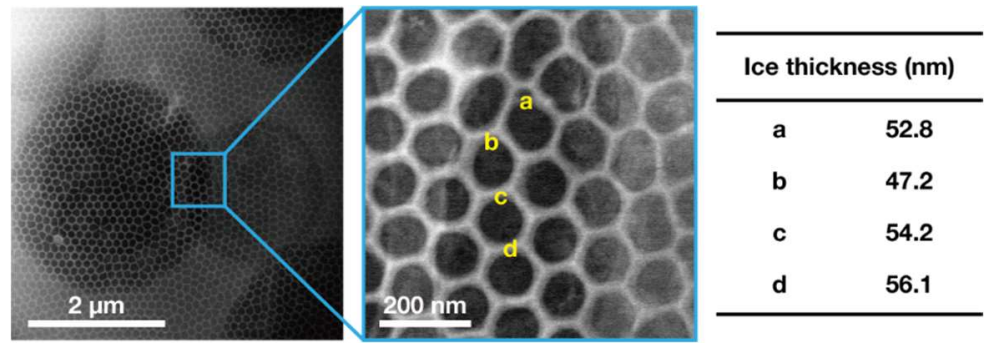

Figure S1. (Left, Middle) STEM images of the multichamber graphene liquid cell (with a 60$\mathrm{nm}$ thick AAO membrane) containing a liquid sample in a cryogenic state. (Right) Ice thickness inside each chamber in overlapping areas of carbon hole, as shown in the STEM images. 
a
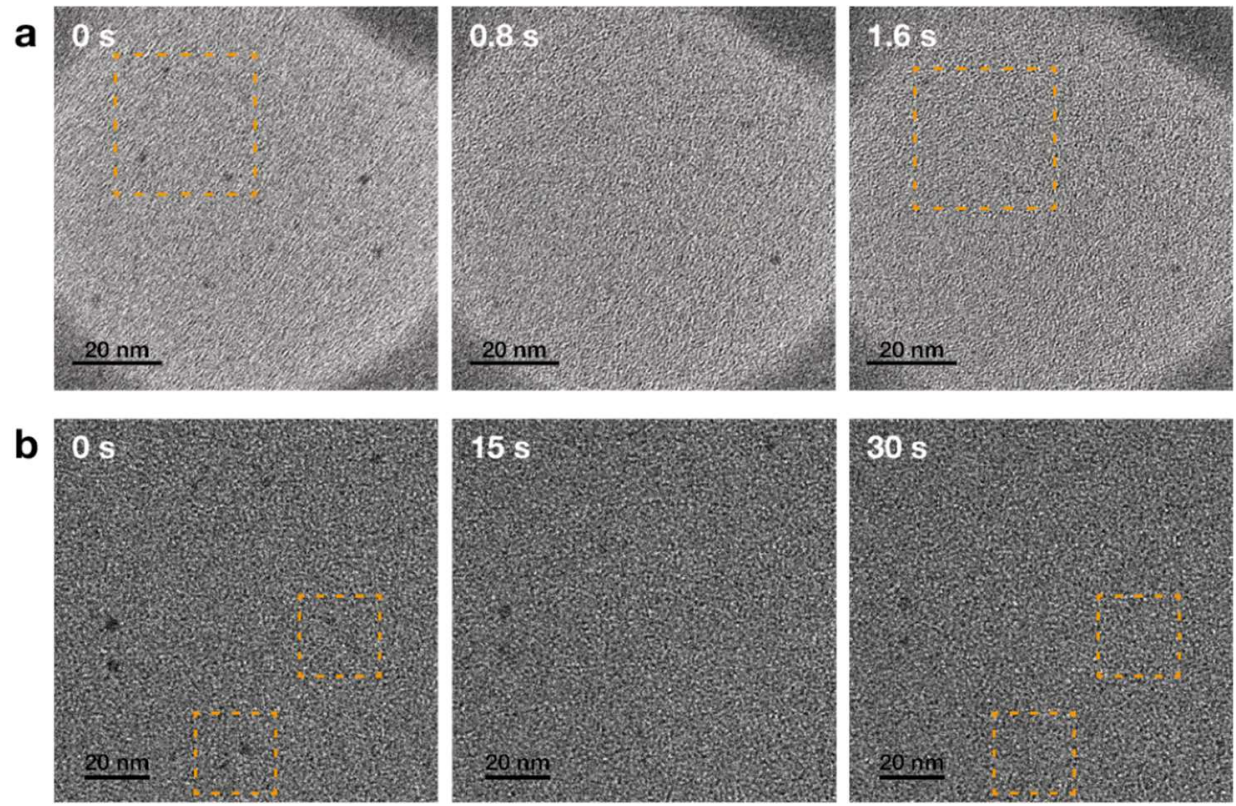

Figure S2. Time-series of TEM images showing the etching of gold nanoparticles at low beam dose rates (a) $\sim 50 \mathrm{e}^{-} \AA^{-2} \mathrm{~s}^{-1}$, (b) $\sim 300 \mathrm{e}^{-} \AA^{-2} \mathrm{~s}^{-1}$. 

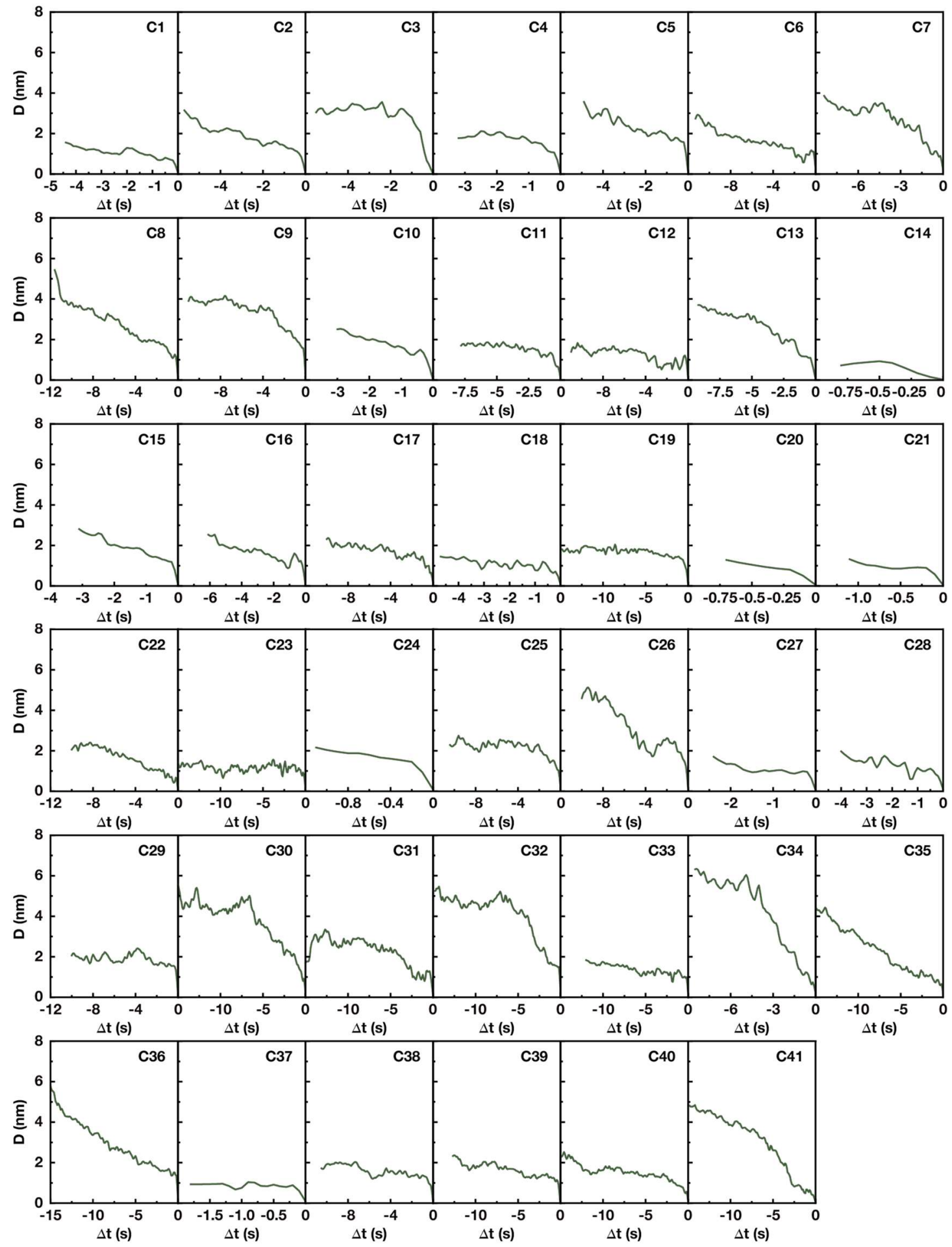

Figure S3. Gap trajectories of 41 tracked pairs of AuNPs/CTAB. 

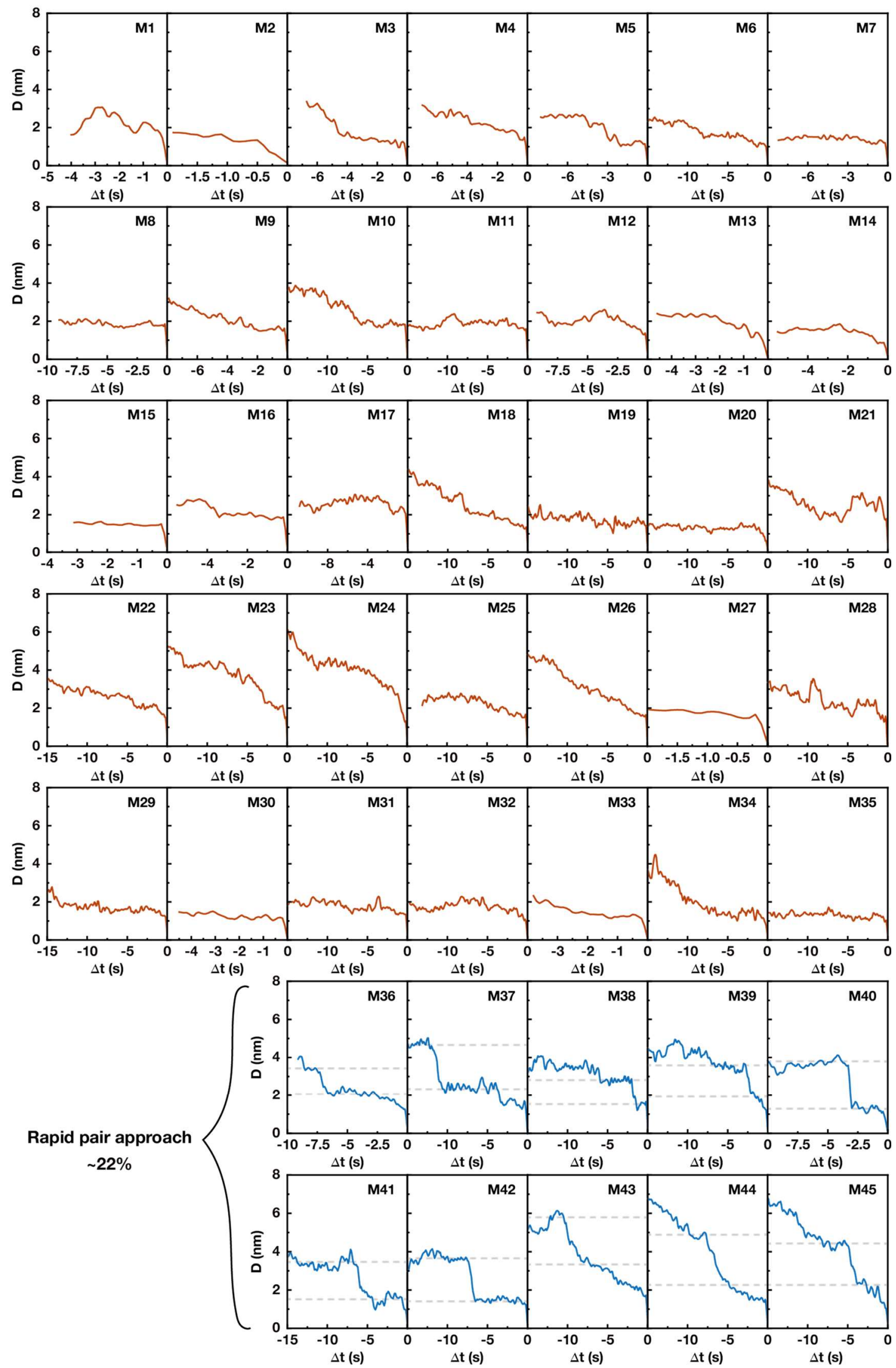

Figure S4. Gap trajectories of 45 tracked pairs of AuNPs/Mixed. Among them, the blue plots demonstrate a rapid pair approach. 

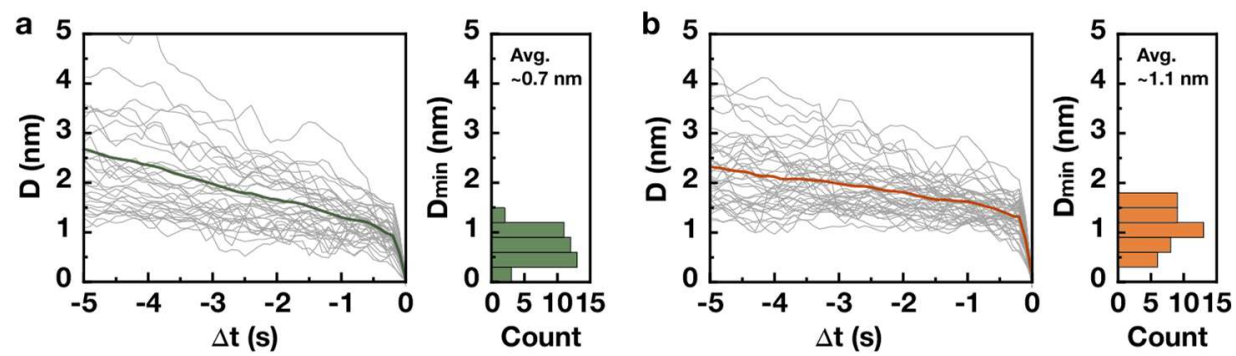

Figure S5. Overlapped gap-trajectories and gap-minimum (at $\Delta t=-0.1 \mathrm{~s}$ ) histograms of (a) the 41 pairs in the AuNPs/CTAB system and (b) the 45 pairs in the AuNPs/Mixed system, before the surface contact. The colored solid thick line represents the mean value of the gap trajectory. 

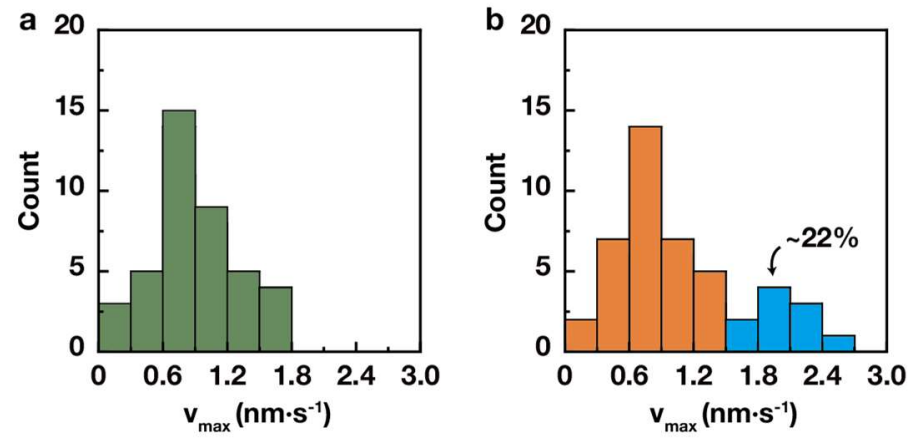

Figure S6. Maximum pairwise approach speed for the pairs in the (a) AuNPs/CTAB and (b) AuNPs/Mixed systems. 

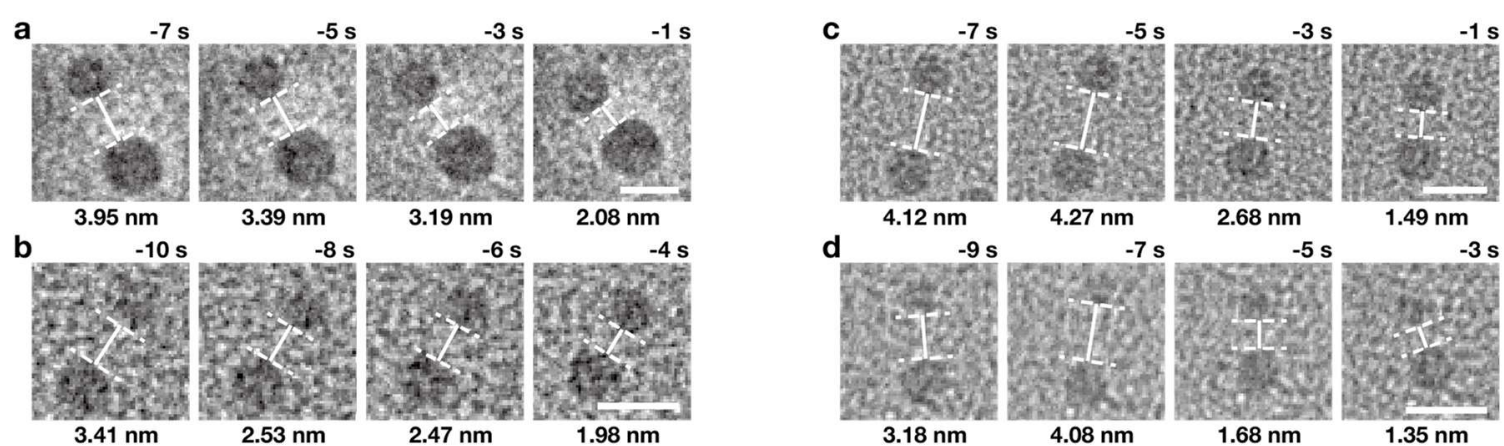

Figure S7. Time-series of TEM images of the approach behaviors of the gold nanoparticles in the $(\mathrm{a}-\mathrm{b})$ AuNPs/CTAB and $(\mathrm{c}-\mathrm{d})$ AuNPs/Mixed systems. In the upper values, time $=\Delta t$. The lower values are the gaps between the nanoparticles (white solid stick) (scale bars, $5 \mathrm{~nm}$ ). The gap-trajectories of the pair in (a), (b), (c), and (d) correspond to the plots in Figures (d), $(\mathrm{e}),(\mathrm{g})$, and $(\mathrm{h})$ in the main text, respectively. 

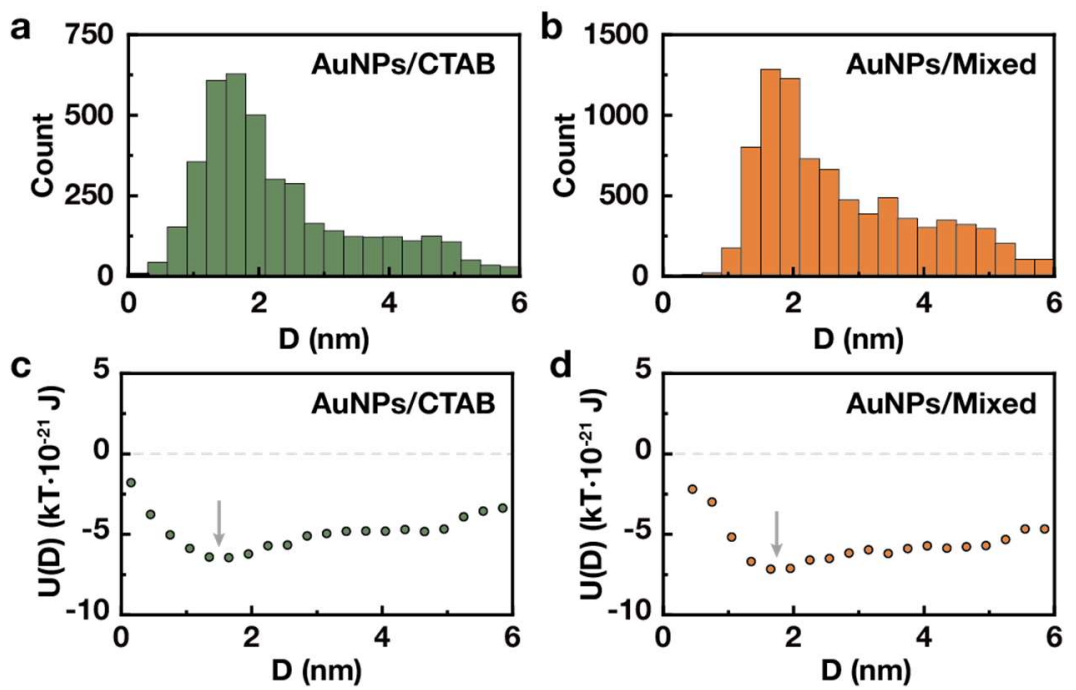

Figure S8. Combined distribution of all pairwise surface separation distances $(D)$ for (a) AuNPs/CTAB and (b) AuNPs/Mixed systems. Pairwise interaction potential as a function of $D$ for (c) AuNPs/CTAB and (d) AuNPs/Mixed systems. The gray arrow indicates the minimum value. 


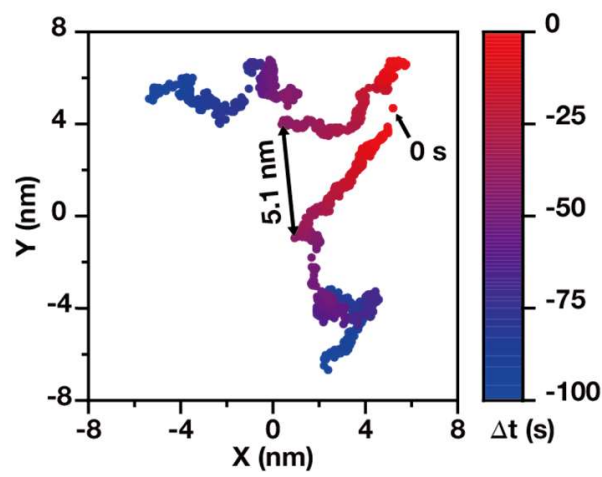

Figure S9. Tracking of pairwise nanoparticle motion for AuNP/Mixed system before the surface contact. $\Delta t$ means $t-t_{0}$ with respect to $t_{0}$, at the instance of contact. 
(111)

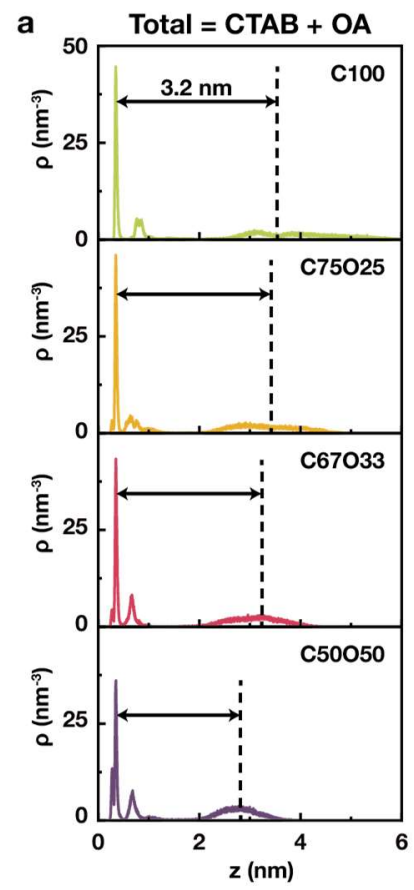

(110)

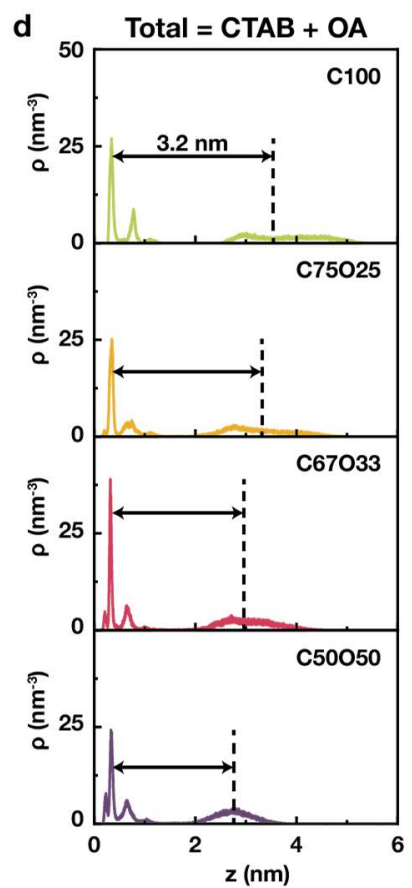

b

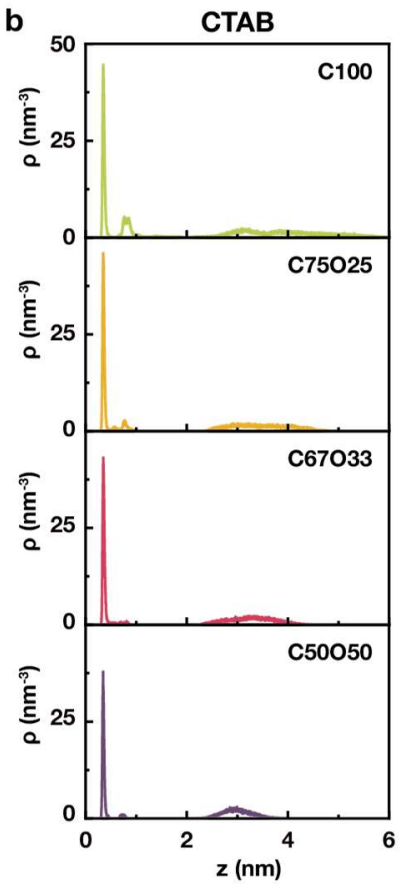

e

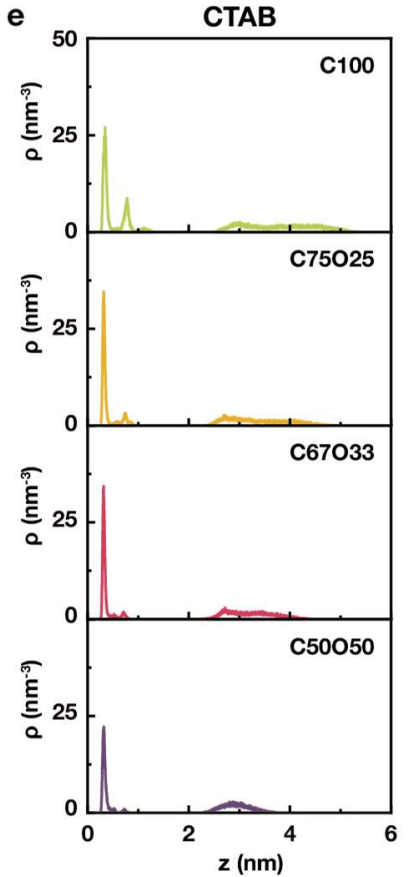

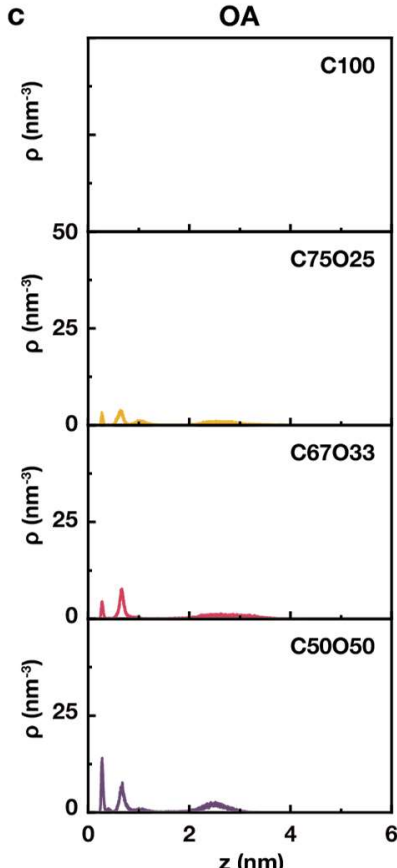

f

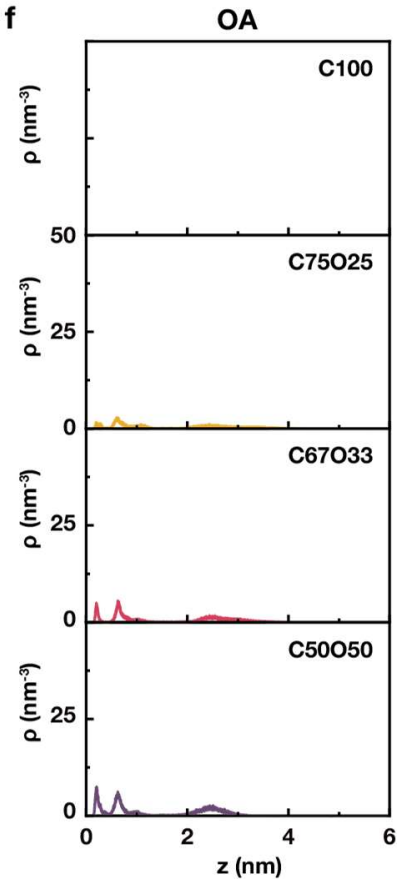

Figure S10. Number density of $\mathrm{N}$ atoms, as a function of the distance, from the gold $(\mathrm{a}-\mathrm{c})$ (111) and (d-f) (110) surfaces. (a), (d) for all the ligand molecules; (b), (e) for only CTAB; (c), (f) for only octylamine (OA), respectively. 

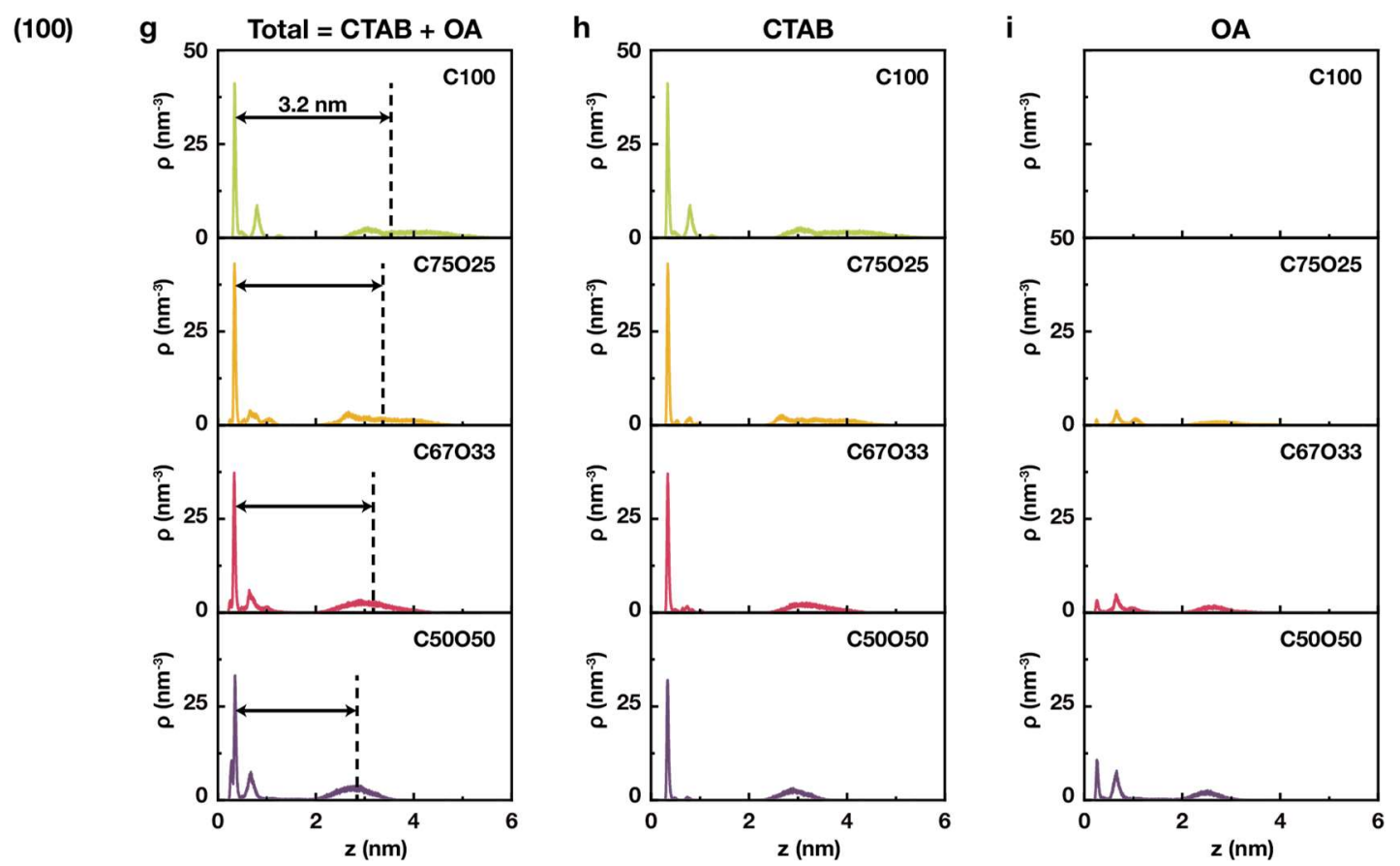

Figure S10. (Continued) Number density of $\mathrm{N}$ atom, as a function of the distance, from the gold (g-i) (100) surfaces. (g) for all ligand molecules; (h) for only CTAB; (i) for only OA, respectively. 

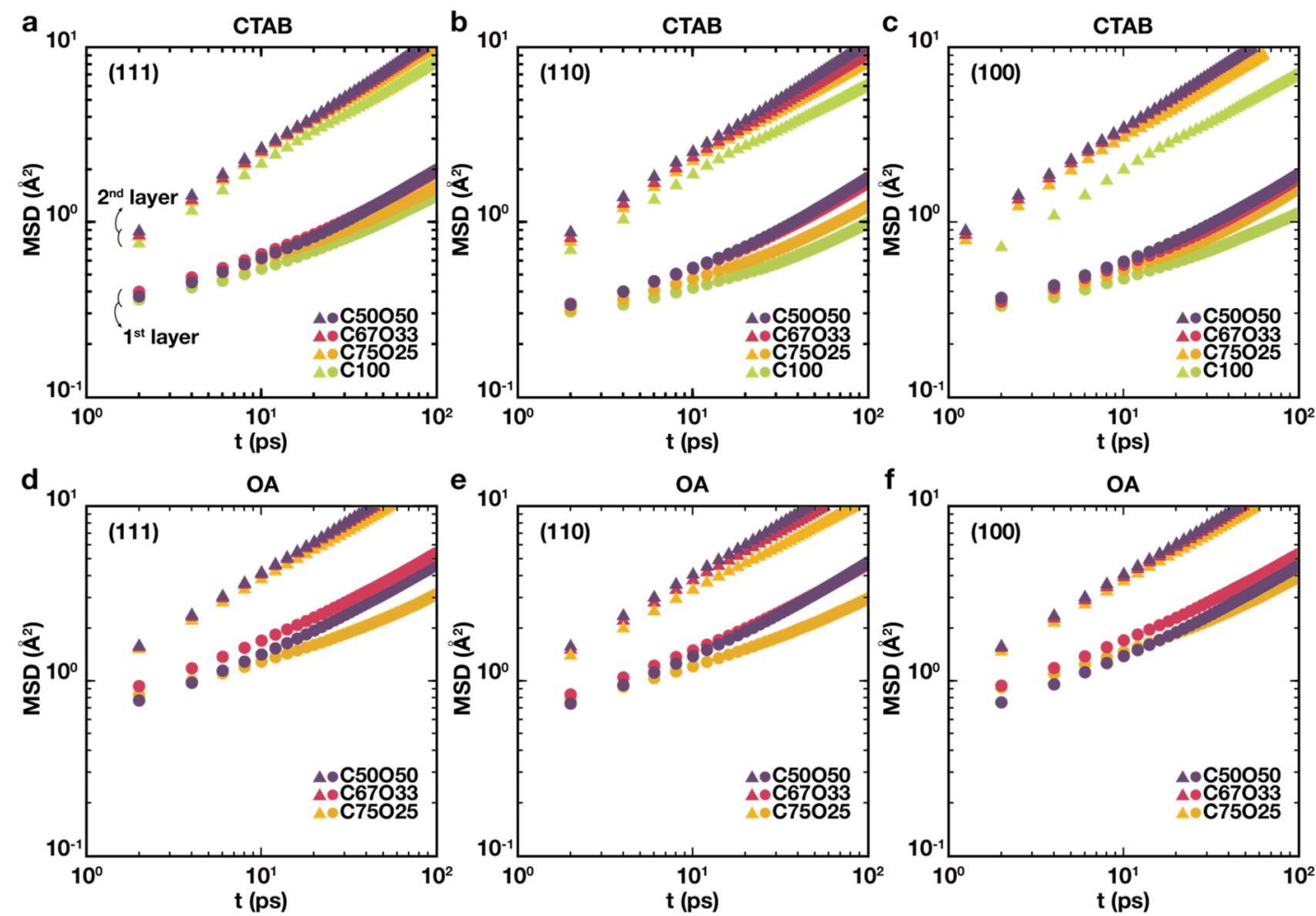

Figure S11. Ensemble-averaged MSD, as a function of time, for $(\mathrm{a}-\mathrm{c})$ only CTAB and $(\mathrm{d}-\mathrm{f})$ only the OA molecules in the ligand shell. (a), (d) on the gold (111) surface; (b), (e) on the gold (110) surface; (c), (f) on the gold (100) surface. The top four plots (triangles) and bottom four plots (circles) of each graph are calculated from the molecules in the second and the first layers of the ligand shell. 


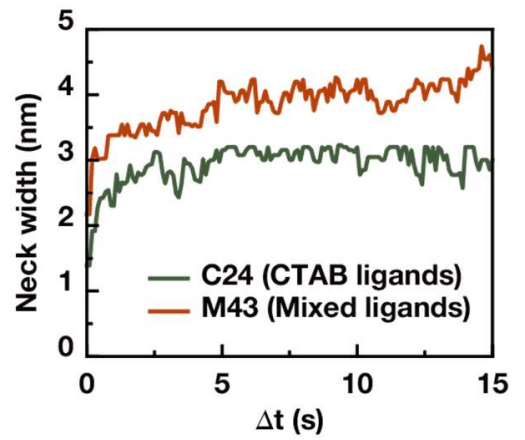

Figure R12. The evolution of the neck width of the representative merged gold nanoparticles. Corresponding time-series of the TEM images are shown in Figures $4 \mathrm{a}$ and $\mathrm{b}$. From the neck evolution as a function of time, the exponent $a$ was estimated by the power laws in the form of $d_{\text {neck }} \sim t^{a}$. The estimated $a$ for the C24 and M43 is 0.29 and 0.36 at the early stage and 0.08 and 0.09 at the late stage, respectively. The results indicate that the neck evolves rapidly just after the contact and later the neck is recovered mainly by surface diffusion ( $a \sim 0.14$, suggested by Kucaynski. ${ }^{20}$ ) in both systems. 


\section{References}

(1) Lim, K.; Bae, Y.; Jeon, S.; Kim, K.; Kim, B. H.; Kim, J.; Kang, S.; Heo, T.; Park, J.; Lee, W. C. A Large-Scale Array of Ordered Graphene-Sandwiched Chambers for Quantitative Liquid-Phase Transmission Electron Microscopy, Adv. Mater. 2020, 2002889.

(2) Regan, W.; Alem, N.; Alemán, B.; Geng, B.; Girit, Ç.; Maserati, L.; Wang, F.; Crommie, M.; Zettl, A. A direct transfer of layer-area graphene. Appl. Phys. Lett. 2010, 96, 113102.

(3) Schneider, N. M.; Norton, M. M.; Mendel, B. J.; Grogan, J. M.; Ross, F. M.; Bau, H. H. Electron-water interactions and implications for liquid cell electron microscopy. J. Phys. Chem. C 2014, 118, 22373-22382.

(4) Huang, J.; MacKerell Jr, A. D., J. CHARMM36 all-atom additive protein force field: Validation based on comparison to NMR data. Comp. Chem. 2013, 34, 2135-2145.

(5) Storm, S.; Jakobtorweihen, S.; Smirnova, I.; Panagiotopoulos, A. Z. Molecular Dynamics Simulation of SDS and CTAB Micellization and Prediction of Partition Equilibria with COSMOmic. Langmuir 2013, 29, 11582-11592.

(6) Plimpton, S. J.; Pollock, R.; Stevens, M. Particle-Mesh Ewald and rRESPA for Parallel Molecular Dynamics Simulations. In PPSC 1997.

(7) Ryckaert, J. P.; Ciccotti, G.; Berendsen, H. J. C. J. Numerical integration of the Cartesian Equations of Motion of a System with Constraints: Molecular Dynamics of n-Alkanes. Comput. Phys. 1977, 23, 327-341.

(8) Plimpton, S. Fast Parallel Algorithms for Short-Range Molecular Dynamics. J. Comp. Phys. 1995, 117, 1-19.

(9) Egerton, R. F. Electron energy-loss spectroscopy in the electron microscope. Springer Science \& Business Media: 2011.

(10) Feja, B.; Aebi, U. Determination of the inelastic mean free path of electrons in vitrified ice layers for on-line thickness measurements by zero-loss imaging. J. Microsc. 1999, 193, 15.

(11) Cho, H. J.; Hyun, J. K.; Kim, J. G.; Jeong, H. S.; Park, H. N.; You, D. J.; Jung, H. S. Measurement of ice thickness on vitreous ice embedded cryo-EM grids: investigation of optimizing condition for visualizing macromolecules. J. Anal. Sci. Technol. 2013, 4, 7.

(12) Rice, W. J.; Cheng, A.; Noble, A. J.; Eng, E. T.; Kim, L. Y.; Carragher, B.; Potter, C. S. Routine determination of ice thickness for cryo-EM grids. J. Struct. Biol. 2018, 204, 38.

(13) Ross, F. M. Liquid Cell Electron Microscopy. Cambridge University Press: 2016.

(14) Grogan, J. M.; Rotkina, L.; Bau, H. H. In situ liquid-cell electron microscopy of colloid aggregation and growth dynamics. Phys. Rev. E 2011, 83, 061405.

(15) Ring, E. A.; de Jonge, N. Video-frequency scanning transmission electron microscopy of moving gold nanoparticles in liquid. Micron 2012, 43, 1078-1084.

(16) Kelly, D. J.; Zhou, M.; Clark, N.; Hamer, M. J.; Lewis, E. A.; Rakowski, A. M.; Haigh, S. J.; Gorbachev, R. V. Nanometer resolution elemental mapping in graphene-based TEM liquid cells. Nano Lett. 2018, 18, 1168-1174.

(17) Verch, A.; Pfaff, M.; de Jonge, N. Exceptionally slow movement of gold nanoparticles at a solid/liquid interface investigated by scanning transmission electron microscopy. Langmuir 2015, 31, 6956-6964. 
(18) Cho, H.; Jones, M. R.; Nguyen, S. C.; Hauwiller, M. R.; Zettl, A.; Alivisatos, A. P. The use of graphene and its derivatives for liquid-phase transmission electron microscopy of radiation-sensitive specimens. Nano Lett. 2017, 17, 414-420.

(19) Zan, R.; Ramasse, Q. M.; Jalil, R.; Georgiou, T.; Bangert, U.; Novoselov, K. S. Control of radiation damage in MoS2 by graphene encapsulation. ACS Nano 2013, 7, 10167-10174.

(20) Kuczynski, G. C. Study of the sintering of glass. J. Appl. Phys. 1949, 20, 1160-1163. 